\title{
EDITORIAL \\ Artificial neural networks for neurosurgical diagnosis, prognosis, and management
}

\author{
Robert E. Harbaugh, MD \\ Department of Neurosurgery, Penn State University, Penn State Hershey Medical Center, Hershey, Pennsylvania
}

$\mathrm{N}$ EUROSURGEONS are faced with a dizzying array of data that need to be analyzed when making clinical decisions about diagnosis, prognosis, and management. Over the last 30 years we have seen the rapid growth in computer processing power and ever-accelerating interest in how to use this computational ability for better data analysis. One aspect of this is whether or not artificial intelligence methods, such as machine learning algorithms and artificial neural networks (ANNs), could improve our capabilities for accurate diagnosis and prognosis and improved patient management. The appeal of ANNs is obvious. They employ diverse types of medical data as inputs and integrate them into categorized outputs. This is, in essence, what clinicians do so the process has some face validity.

The paper by Hale and colleagues in this issue of $\mathrm{Neu}$ rosurgical Focus evaluates the ability of an ANN to accurately predict 6-month outcomes in pediatric patients with traumatic brain injury (TBI). ${ }^{7}$ They compare their ANN analysis to conventional statistical models such as multivariable analysis and to predictive models based on CT classification schemes. In this study the ANN approach outperformed CT classification schemes and traditional statistical analysis for accurately predicting 6-month outcome. I will briefly discuss the evolution of predicting the outcome of patients with TBI and delineate the ANN approach for medical diagnosis, prognosis, and management.

The recent evolution of prognostication in patients with head injury is fascinating. In 1974 Teasdale and Jennett published their landmark article on the use of a prognostic scale based solely on clinical data. ${ }^{12}$ As advanced brain imaging became increasingly important in the diagnosis and prognosis of neurosurgical diseases and disorders new prognostic schemes that incorporated such data were proposed..$^{8}$ Neurosurgeons in 2018 must learn to determine the prognosis of head trauma patients by considering many, many input variables such as the history of the patient's present illness, patient age, comorbidities and other aspects of the past medical history, findings on physical and neurological examinations, the results of advanced imaging studies and laboratory assessments, physiological monitoring data, and so on. These data are fed into the "black box" of the individual clinician's ability to integrate this information and use it to create a mental model based on the input data and a wealth of other data obtained through formal education and personal experience. The output of this system is a mental model that is used to predict the patient's clinical course and outcome. The model is regularly updated as additional data (e.g., uncontrolled intracranial pressure, hypoxia, hypotension, or infectious complications) become available during the course of the patient's treatment.

In 1988, the concept of using artificial intelligence in medical diagnosis was first reported in the pioneering work of Szolovits and colleagues. ${ }^{11}$ Since then, review papers and papers dealing with the use of one type of artificial intelligence approach, ANNs, for diagnosing a wide

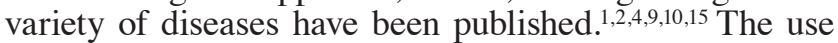
of ANNs in determining prognosis has also been reported, ${ }^{13,14}$ including an earlier paper not cited by the authors on the use of machine learning for predicting the outcome of pediatric head injury patients. ${ }^{6}$ The following description of ANNs will strive to be free of jargon and mathematics. For the engineers, mathematicians, and masochists in the audience a more formal review of the mathematical structure underlying ANNs can be found in the papers of Amato et al. and Basheer and Hajmeer., ${ }^{3,5}$

An ANN is a mathematical representation of an actual neural network that shares a neural network's ability to learn and generalize. Like true neural networks, ANNs can model highly nonlinear systems in which the relationships among many variables are unknown. The network is formed by a series of nodes (analogous to neurons) organized in layers. Each node in a layer is connected with each node in the next layer through a weighted connection. The weighting determines the strength of the connection. 
The ANN has an input layer, one or more hidden layers, and an output layer. The number of nodes in a layer and the number of layers depends on the complexity of the system being studied. The mathematical process through which the network learns can be ignored by the clinician. Because of this the ANN is often referred to as a "black box" process. ${ }^{3}$

The network learns from a series of examples in a training database. The database is a matrix of data concerning patients for whom the diagnosis or prognosis is already known. Each row of the matrix refers to one patient, with columns of input data and a column for the output (clinical outcome). Learning is achieved by iteratively changing the values of the connection weights between nodes according to mathematical rules in the training algorithm. The neural network is trained using a suitable database of example cases. An example is provided by one patient for whom the values for the input features and the outcome are known. The quality of training and the predictive ability of the network depend on the reliability of the data and the number of examples in the database used for training. This training database must contain a sufficient number of examples to allow the network to extract the structure hidden in the data set and then use this information gained from the learning process to generalize the rule to new cases. I would submit that this is exactly what resident training does as well.

After determining the optimal network architecture, the ANN is verified by using examples not used in the training process. As the final step, the ANN output must be tested in clinical practice to determine its accuracy. These new patients can also be added to the examples in the training database. Machine learning algorithms are iterative, and as more and more reliable examples are analyzed the predictive accuracy of the ANN improves. Thus, ANNs mimic the activity of the neurosurgeon by using a wide variety of input data processed in the context of previous training. As computational power increases and the training data become more reliable and complete the output of the system should steadily improve.

ANNs are becoming increasingly powerful tools for assisting physicians in diagnosis and prognosis of disease. They have the ability to process large amounts of data without ignoring relevant information and may be able to assist not only in diagnosis and prognosis but also in the management of complex patients. In the Neurocritical Care Unit (NCCU) at the Penn State Hershey Medical Center we are assessing the ability of machine learning algorithms to aid in the management of neurocritical care patients. At present we gather an enormous amount of data on each NCCU patient including clinical history and examination data, imaging data, extensive physiological and neurophysiological monitoring data, laboratory data, etc. We use the traditional "black box" approach of having our neurosurgeon neurointensivists integrate this tsunami of data in light of our previous training and experience to create a mental model of the patient's present state. This model is employed to determine which interventions should be used to avoid a deleterious change in the state of the patient and thus to maximize the patient's chances for a good recovery. I believe that the use of machine learn- ing algorithms will be able to help us refine our ability to predict the future state of the patient from the present state and to suggest interventions that improve the chances for a favorable outcome. Artificial intelligence will become a valuable adjunct to clinical judgement for improving neurosurgical diagnosis, prognosis, and management.

https://thejns.org/doi/abs/10.3171/2018.8.FOCUS18438

\section{References}

1. Ahmed F: Artificial neural networks for diagnosis and survival prediction in colon cancer. Mol Cancer 4:29, 2005

2. Alkim E, Gürbüz E, Kiliç E: A fast and adaptive automated disease diagnosis method with an innovative neural network model. Neural Netw 33:88-96, 2012

3. Amato F, López A, Peña-Méndez EM, Vaňhara P, Hampl A, Havel J: Artificial neural networks in medical diagnosis. J Appl Biomed 11:47-58, 2013

4. Bartosch-Härlid A, Andersson B, Aho U, Nilsson J, Andersson R: Artificial neural networks in pancreatic disease. Br J Surg 95:817-826, 2008

5. Basheer I, Hajmeer M: Artificial neural networks: fundamentals, computing, design, and application. J Microbiol Meth 43:3-31, 2000

6. Chong SL, Liu N, Barbier S, Ong MEH: Predictive modeling in pediatric traumatic brain injury using machine learning. BMC Med Res Methodol 15:22, 2015

7. Hale AT, Stonko DP, Brown A, Lim J, Voce DJ, Gannon SR, et al: Machine-learning analysis outperforms conventional statistical models and CT classification systems in predicting 6 -month outcomes in pediatric patients sustaining traumatic brain injury. Neurosurg Focus 45(5):E2, 2018

8. Marshall LF, Marshall SB, Klauber MR, Van Berkum Clark $\mathrm{M}$, Eisenberg H, Jane JA, et al: The diagnosis of head injury requires a classification based on computed axial tomography. J Neurotrauma 9 (Suppl 1):S287-S292, 1992

9. Penny W, Frost D: Neural networks in clinical medicine. Med Decis Making 16:386-398, 1996

10. Siristatidis C, Chrelias C, Pouliakis A, Katsimanis E, Kassanos D: Artificial neural networks in gyneacological diseases: current and potential future applications. Med Sci Monit 16:RA231-RA236, 2010

11. Szolovits P, Patil RS, Schwartz W: Artificial intelligence in medical diagnosis. Ann Intern Med 108:80-87, 1988

12. Teasdale G, Jennett B: Assessment of coma and impaired consciousness. A practical scale. Lancet 2:81-84, 1974

13. Walczak S: Artificial neural network medical decision support tool: predicting transfusion requirements of ER patients. IEEE Trans Inf Technol Biomed 9:468-474, 2005

14. Wise ES, Stonko DP, Glaser ZA, Garcia KL, Huang JJ, Kim JS, et al: Prediction of prolonged ventilation after coronary artery bypass grafting: data from an artificial neural network. Heart Surg Forum 20:E007-E014, 2017

15. Yoldaş Ö, Tez M, Karaca T: Artificial neural networks in the diagnosis of acute appendicitis. Am J Emerg Med 30:12451247,2012

\section{Disclosures}

The author reports no conflict of interest.

\section{Correspondence}

Robert E. Harbaugh: reh21@psu.edu.

\section{INCLUDE WHEN CITING}

DOI: 10.3171/2018.8.FOCUS18438. 